\title{
Phase equilibria in a model of low-salt suspensions of charged colloids
}

\author{
René van Roij and Robert Evans \\ H H Wills Physics Laboratory, Royal Fort, University of Bristol, Bristol BS8 1TL, UK
}

Received 21 July 1999

\begin{abstract}
We calculate phase diagrams of low-salt suspensions of charged colloidal particles using a recently proposed effective one-component Hamiltonian for the colloids (van Roij R, Dijkstra M and Hansen J-P 1999 Phys. Rev. E 59 2010). This Hamiltonian consists of the purely repulsive pairwise Derjaguin, Landau, Verwey, and Overbeek (DLVO) potential and densitydependent volume terms. The latter play a crucial role in driving phase transitions at salt concentrations of the order of $10 \mu \mathrm{M}$ or lower. We find phase diagrams that exhibit gas-liquid coexistence with upper and lower critical points, and, for sufficiently large surface charge densities, upper and lower gas-liquid-solid triple points. A connection between the Debye-Hückel theory for simple electrolytes and the DLVO theory for colloidal suspensions is made.
\end{abstract}

\section{Introduction}

More than 125 years ago, Van der Waals argued that gas-liquid coexistence in simple fluids can be attributed to the interplay between short-ranged (steric) repulsions and long-ranged (dispersive) attractions between atoms or molecules [1]. He showed that it is thermodynamically 'favourable' for a classical fluid at intermediate density to phase separate into a dilute gas (with a high entropy) and a dense liquid (with a low internal energy), if the temperature is sufficiently low, i.e. if the attractive interactions are sufficiently strong compared to the thermal energy $k_{B} T$ [2]. Van der Waals' ideas have been much refined and quantified in the course of the 20th century, but his insight that the liquid phase in simple fluids is stabilized by the cohesive energy arising from long-ranged attractions and prevented from collapse by short-ranged repulsions has long been accepted.

The interplay between short-ranged repulsions and long-ranged attractions has also been a central theme in colloid science since the 1930s, when it was realized that the stability of a colloidal suspension is determined primarily by the competition between double-layer repulsions and dispersive attractions [3-5]. The theory developed by Derjaguin, Landau, Verwey, and Overbeek (DLVO) describes the effective interactions between two colloidal particles in a 1:1 electrolyte of salt concentration $n_{+}=n_{-}=n_{s}$ and solvent dielectric constant $\epsilon$ as a sum of a screened Coulomb repulsion and a Van der Waals-London-Hamaker attraction [3-6]. It predicts that the amplitude of the pairwise repulsion is proportional to the product of the electric charges of the two colloids, whereas the range is given by the Debye screening length $\lambda_{D}=1 / \kappa_{D}$ of the electrolyte, with $\kappa_{D}^{2}=8 \pi e^{2} n_{s} / \epsilon k_{B} T$, $e$ being the unit charge. For water at room temperature one finds, for salt concentrations $n_{s}=1 \mathrm{M}, 1 \mathrm{mM}$, and $1 \mu \mathrm{M}$, that $\lambda_{D} \simeq 0.3,10,300 \mathrm{~nm}$, respectively. The amplitude of the colloid-colloid attraction is determined by the material-dependent Hamaker constant $A \simeq 10^{-18}-10^{-20} \mathrm{~J}$, and 
its range extends typically to a few nanometres from the surface of the colloidal particles. The competition between repulsion and attraction in this DLVO pair interaction depends strongly on $n_{s}$, and this explains, at least qualitatively, several experimental observations. At high salt concentrations $n_{s} \simeq 1 \mathrm{M}$, where $\lambda_{D} \ll 1 \mathrm{~nm}$, and hence attraction dominates over repulsion according to the DLVO theory, suspensions are indeed unstable and show irreversible flocculation 'into the primary minimum' of the pair potential at contact [7]. At intermediate salt concentrations $n_{s} \simeq 10 \mathrm{mM}$, where $\lambda_{D} \simeq 1 \mathrm{~nm}$, the attraction balances the repulsion and reversible 'gas-liquid' coexistence is possible [6], whereas the regime of colloidal stability at lower salt concentrations, with $\lambda_{D} \gg 1 \mathrm{~nm}$, corresponds to dominant repulsions. Many more experimentally observed phenomena in colloidal suspensions have been explained on the basis of the DLVO theory, which has become a well-established cornerstone of colloid science.

However, since the early 1980s several experimental results have been reported that seem to be qualitatively at odds with the DLVO theory. These experiments, performed invariably at extremely low salt concentrations $n_{s} \simeq 1-10 \mu \mathrm{M}$, reveal strong evidence for coexistence of a very dilute colloidal gas phase with a much denser colloidal liquid or crystal [8-11]. By analogy with Van der Waals' line of reasoning in the case of simple fluids, one expects that gas-liquid and gas-solid coexistence in colloidal suspensions should require long-ranged attractions. Since we have seen above that the dispersive attraction is completely masked by the electric double-layer repulsion in the extreme low-salt regime $n_{s} \simeq 1-10 \mu \mathrm{M}$, one cannot explain the observations by invoking the Van der Waals-London-Hamaker interaction. Instead it was argued that the experiments should be interpreted as evidence for the existence of electrostatic attractions between colloids with charge of the same sign. In other words, the low-salt experiments seem to invalidate the well-established DLVO theory!

The earliest attempt to explain these low-salt experiments was due to Sogami in 1983 [12] and Sogami and Ise in 1984 [13]. These authors argued that one should consider not the Helmholtz free energy of the microions-which leads to the DLVO theory-but instead the corresponding Gibbs free energy (of the isothermal isobaric ensemble) when determining the effective colloid-colloid interaction. Their analysis revealed that the Gibbs pair potential between the colloids exhibits a long-range attraction of electrostatic origin that could be strong enough to drive gas-liquid or gas-solid phase separation in low-salt suspensions [12-14]. However, the fundamentals of the Sogami-Ise theory have remained at the centre of intense debate $[15,16]$. It was shown by Woodward in 1988 , for instance, that the inhomogeneity of the microions (due to the presence of the charged colloids) invalidates the key assumption in the Sogami-Ise theory that the Gibbs free energy is a first-order homogeneous function of the particle numbers [16]. More recently many other attempts to describe net attractions between like-charged colloidal particles have appeared in the literature [17-19]. Most of these are based on the influence of microion charge correlations or colloid charge fluctuations, which are not taken into account in the (mean-field-like) DLVO theory. In general the resulting attractive contribution to the colloid-colloid pair interaction arising from correlation effects is rather weak and short ranged, and often requires the microions to be multivalent. It also appears that the attractions, if present, are suppressed for colloidal spheres as compared to discs [20]. The recently proposed Coulombic depletion mechanism [21], by analogy with the depletion effect in colloid-polymer mixtures, also gives rise to effective colloid-colloid attraction at high Coulomb coupling. However, we are not aware of a study (with the exception of reference [19]) that shows that any of these fluctuation, correlation, or depletion effects can explain the phase behaviour observed in the low-salt suspensions.

It is important to realize that knowledge of the effective interactions between colloids is only a first (albeit important) step in the determination the phase behaviour of a colloidal suspension. A popular route towards the study of the phase behaviour starts with the study 
the effective potential between two colloidal particles in the presence of a bath of microions, proceeds via the assumption that the interaction Hamiltonian of the colloidal suspension is then simply the sum of this pair potential between all pairs of colloidal particles, and concludes with the calculation of an appropriate thermodynamic potential, e.g. the Helmholtz free energy, corresponding to that Hamiltonian. Clearly, the DLVO pair potential has often been used successfully in this scheme. In this paper we follow a more systematic route towards the phase diagram of a model for a colloidal suspension by calculating the total free energy of a three-component mixture of highly charged hard spheres and monovalent positive and negative point ions. The effective Hamiltonian of the colloidal particles, obtained by integrating out the degrees of freedom of the point ions, is an intermediate result of this procedure. Despite the fact that our calculations are at the linearized Poisson-Boltzmann level, in the spirit of the original calculations in references $[3,4]$, the resulting effective one-component Hamiltonian goes beyond the often-employed pairwise DLVO approximation in two important respects. First, we find that the electrostatic screening is not only determined by the thermodynamic state of the bath of microions but also by the colloid density; it reduces to that of the bath in the limit of high salt concentration or low colloid density. Second, we find important nonpairwise contributions which were not incorporated in early studies, but which do appear in more recent studies, e.g. references $[18,19,28,29]$. At high salt concentrations the present theory is equivalent to the standard DLVO theory, whereas at extremely low salt concentrations it predicts gas-liquid and gas-solid coexistence. Thus the theory provides, at least qualitatively, an explanation for some puzzling and controversial experimental observations. Moreover, the theory also connects the DLVO theory for colloidal suspensions to another classical linearized Poisson-Boltzmann theory, namely the Debye-Hückel theory for simple electrolytes. The latter is well known to predict gas-liquid coexistence at low temperatures. The required cohesive energy that stabilizes the dense liquid phase of simple electrolytes is provided by a combination of charge neutrality and correlations: every ion is surrounded by a cloud of opposite charge. We argue that the recently observed gas-liquid and gas-solid coexistence in colloidal suspensions is driven by a mechanism equivalent to that in simple electrolytes, and not, as has often been claimed, by pairwise electrostatic attraction between like-charged colloidal particles. We also show that the charge and size asymmetry of the colloidal suspension enriches the phase diagram compared to that of simple electrolytes, i.e. the asymmetry gives rise to closed-loop gas-liquid and gas-solid coexistence, with upper and lower critical and triple points. The approach that we apply is that put forward in references [22,23]. By extending this to a larger class of system parameters, new features of the phase equilibria are revealed. Although our presentation is self-contained, we refer to reference [23] for many of the technical details.

\section{Model and effective Hamiltonian}

We consider a three-component mixture consisting of (i) $N_{c}$ colloidal spheres of diameter $D=2 R>0$ and charge $-Z e$ (with $Z \gg 1$ ) distributed homogeneously over the surface, (ii) $N_{s}$ point ions of charge $-e$, and (iii) $N_{s}+Z N_{c}$ point ions of charge $+e$, all suspended in a structureless medium of dielectric constant $\epsilon$ and total volume $V$ at a constant temperature $T$. This mixture is electrically neutral. Having in mind the low-salt regime discussed above, we simply ignore the dispersion interactions between any of the three components, and restrict attention to Coulombic and steric hard-core interactions. Using the label ' $\mu$ ' to denote collectively all positive and negative microions, we consider the total Hamiltonian $H=H_{c}+H_{\mu}+H_{c \mu}$, where the bare colloid and microion Hamiltonians are $H_{c}$ and $H_{\mu}$, respectively, and the colloid-microion interaction term is $H_{c \mu}$. The final goal is to calculate 
the Helmholtz free energy $F\left(N_{c}, N_{s}, V\right)$ of this system, and, hence, the phase behaviour. Formally, one can write the canonical partition function of the mixture as

$$
\exp [-\beta F]=\operatorname{Tr}_{c} \operatorname{Tr}_{\mu} \exp [-\beta H] \equiv \operatorname{Tr}_{c} \exp \left[-\beta H_{c}^{\text {eff }}\right]
$$

where the traces $\operatorname{Tr}_{c}$ and $\operatorname{Tr}_{\mu}$ are short for the canonical phase-space integrals over the colloidal and microion degrees of freedom, respectively. The intermediate result of interest, $H_{c}^{\text {eff }}$, as defined in equation (1), is called the effective one-component colloid Hamiltonian, because of the analogy with the relation between Hamiltonian and free energy in a one-component system. Given that the bare colloid Hamiltonian $H_{c}$ is independent of the coordinates of the microions, it follows directly from equation (1) that

$$
H_{c}^{\mathrm{eff}}=H_{c}+F^{\prime}
$$

with

$$
\exp \left[-\beta F^{\prime}\right]=\operatorname{Tr}_{\mu} \exp \left[-\beta\left(H_{\mu}+H_{c \mu}\right)\right] .
$$

Clearly, $F^{\prime}$ is the Helmholtz free energy of an inhomogeneous fluid of the microions in the external field of colloids at fixed positions $\boldsymbol{R}_{j}$ with $j=1, \ldots, N_{c}$, i.e. $F^{\prime}=F^{\prime}(\{\boldsymbol{R}\})$. The formalism described in equations (1)-(3) is well known in the theory of liquid metals - where one integrates out the electrons to obtain effective interactions between pseudoatoms [2, 24]. It was also used in reference [25] to describe salt-free suspensions of charged colloids.

In reference [23] we calculated $F^{\prime}$ within density functional theory, thus treating the microion species at the level of their density profiles instead of their individual coordinates $[25,26]$. The approximate functional used in reference [23] ignores microion-microion correlations, and treats the electrostatics at the linearized Poisson-Boltzmann level. This functional is minimized by microion profiles that describe electric double layers surrounding the colloidal particles. The double-layer thickness is found to be governed by the screening length $1 / \kappa$, with the screening parameter $\kappa$ now given by

$$
\kappa^{2}=\frac{4 \pi e^{2}\left(2 n_{s}+Z n_{c}\right)}{\epsilon k_{B} T}
$$

where the colloid density $n_{c}=N_{c} / V$ and the salt concentration $n_{s}=N_{s} / V$. This definition of $\kappa$ only coincides with that of $\kappa_{D}$ of the electrolyte, mentioned above, in the dilute colloid regime $n_{c} \rightarrow 0$, the regime of interest in the determination of the interactions between a single pair of colloids. At finite colloid density $n_{c}$, however, the counterions (of number density $Z n_{c}$ ) also contribute to the screening, at least within the present theoretical description [27]. Obviously, this counterion contribution is negligible, even for finite $n_{c}$, in a regime of sufficiently high salt concentrations $n_{s} \gg Z n_{c}$. The value of $F^{\prime}$, i.e. the minimum value of the functional, follows from inserting the equilibrium (electric double-layer) profiles into the functional. Insertion of the resulting $F^{\prime}$ into equation (2) yields, as was shown explicitly in reference [23], the effective one-component colloid Hamiltonian with the form

$$
H_{c}^{\text {eff }}=H_{\text {DLVO }}(\{\boldsymbol{R}\})+F_{0}\left(n_{c}, n_{s}\right) .
$$

Here $H_{\text {DLVO }}$ depends on the instantaneous set of coordinates of the colloidal particles $\{\boldsymbol{R}\}$, whereas the so-called volume term $F_{0}$ is independent of the coordinates but dependent on the densities $n_{c}$ and $n_{s}$, i.e.

$$
H_{\mathrm{DLVO}}=K_{c}+\sum_{i<j=1}^{N_{c}}\left[v_{\mathrm{HS}}\left(R_{i j}\right)+\frac{Z^{2} e^{2}}{\epsilon}\left(\frac{\exp [\kappa R]}{1+\kappa R}\right)^{2} \frac{\exp \left[-\kappa R_{i j}\right]}{R_{i j}}\right]
$$

with $K_{c}$ the (trivial) classical kinetic energy of the colloids, $v_{\mathrm{HS}}$ the hard-sphere potential, $R_{i j}=\left|\boldsymbol{R}_{i}-\boldsymbol{R}_{j}\right|$ the colloid-colloid separation, $\kappa$ as defined in equation (4), and the 
volume term

$$
\begin{aligned}
\frac{F_{0}\left(n_{c}, n_{s}\right)}{V}= & k_{B} T\left[n_{+}\left(\ln n_{+}-1\right)+n_{-}\left(\ln n_{-}-1\right)+\frac{\eta}{1-\eta} \frac{2 n_{+} n_{-}}{n_{+}+n_{-}}\right] \\
& -\frac{Z^{2} e^{2}}{2 \epsilon(R+1 / \kappa)} n_{c}-\frac{1}{2} \frac{4 \pi Z^{2} e^{2}}{\epsilon \kappa^{2}} n_{c}^{2}
\end{aligned}
$$

with microion densities $n_{-}=n_{s}, n_{+}=n_{s}+Z n_{c}$, and colloid packing fraction $\eta=(4 \pi / 3) n_{c} R^{3}$. One recognizes that $H_{\text {DLVO }}$ is the one-component DLVO Hamiltonian describing the pairwise screened Coulomb repulsions between 'dressed' colloid particles, i.e. the colloids with their double layers. The volume term $F_{0}$, which is usually not considered (references $[18,19,28,29]$ are exceptions), is acquired in the mapping of the original three-component system onto an effective one-component system. The first two terms of equation (7) account for the (everpresent) ideal-gas contributions of the positive and negative microions, and the third for their excluded-volume interactions with the colloids (at packing fraction $\eta$ ). This excluded-volume term is merely the approximation that results from the simple quadratic functional employed in reference [23]; it might be improved upon in an ad hoc fashion by using scaled-particle expressions for the free volume as in the theory of colloid-polymer mixtures [30], or by using ideas for including steric effects in electrolytes as discussed in reference [31]. Here we are content to employ the present expression since it does capture the important feature of monotonic increase as a function of $\eta$. The fourth and fifth terms of equation (7) describe terms of electrostatic origin. The fourth is, up to the factor $n_{c}$, of the order of the Coulomb energy arising from a charge $-Z e$ (on a colloidal particle) and a charge $+Z e$ at a separation $R+1 / \kappa$ (the double-layer charge and thickness, respectively). This term can therefore be interpreted as the 'internal (free) energy' of the dressed colloids per unit volume. The fifth term of equation (7) is the free energy associated with the (positive) double layers in the screened mean field of other (negative) colloids; it largely cancels the mean-field contribution from the colloid-colloid repulsion described by $F_{\mathrm{DLVO}}$. This cancellation was discussed in reference [23] and will be illustrated explicitly below. The presence of the two electrostatic terms in $F_{0}$ is a reminder that the entities which exhibit DLVO pair interactions are not structureless particles but composite ones: a bare colloid of charge $-Z e$ and a surrounding cloud of microions with a compensating net charge $+Z e$.

\section{Total free energy and phase diagrams}

The form (5) of the effective Hamiltonian leads, using (1), to the following approximation for the total Helmholtz free energy of the suspension:

$$
F\left(N_{c}, N_{s}, V\right)=F_{\mathrm{DLVO}}\left(N_{c}, V ; \kappa\right)+F_{0}\left(n_{c}, n_{s}, V\right)
$$

where $F_{\mathrm{DLVO}}$ is the Helmholtz free energy of a one-component fluid with the $\kappa$-dependent Hamiltonian $H_{\mathrm{DLVO}}$, i.e. $\exp \left[-\beta F_{\mathrm{DLVO}}\right]=\operatorname{Tr}_{c} \exp \left[-\beta H_{\mathrm{DLVO}}\right]$. Many accurate and reliable approximations have been developed over the years to estimate the free energy of onecomponent fluids [2]. Our present calculations of $F_{\text {DLVO }}$ are based on the Gibbs-Bogoliubov inequality, with a hard-sphere fluid and the Einstein crystal as reference systems for the colloidal fluid and solid phase, respectively $[2,23,32]$. Once $F$ is known, the chemical potentials of salt and colloids, as well as the osmotic pressure of the suspension, follow directly from (numerical) differentiation [23]. The phase boundaries, determined by equating the chemical potentials and pressure in two coexisting phases, can then be calculated explicitly for given colloidal charge $Z$ and hard-core diameter $D$, dielectric constant $\epsilon$, and temperature $T$. It is easily checked that the last two variables only occur in a particular combination with the unit charge 
$e$. This is the so-called Bjerrum length $\lambda_{B}=e^{2} / \epsilon k_{B} T$, which, at room temperature, takes values $\lambda_{B} \simeq 0.72,2.3$, and $4.1 \mathrm{~nm}$ for the suspending media water, ethanol, and pentanol, respectively [33].

In this contribution we present phase diagrams in the $\eta-n_{s}$ representation for suspensions of charged colloids at room temperature in ethanol, i.e. $\lambda_{B}=2.3 \mathrm{~nm}$. In figures 1 (a)-1(c) we show three of such phase diagrams for colloids of total charge number $Z=500$, and increasing diameters $D=300,350$, and $380 \mathrm{~nm}$, respectively. We distinguish the disordered fluid (F) and ordered crystalline (FCC) phase, as well as the dilute gas (G) and denser liquid (L) phase. The terms 'disordered' and 'ordered' refer to the structure of the colloidal particles; the microions form double layers around the colloidal particles but do not crystallize themselves (as, e.g., in a Wigner crystal). Also the terms 'dilute' and 'dense' refer to the colloid density. The full curves in figure 1 represent the phase boundaries. The F-FCC phase boundary is primarily driven by the repulsive interactions, i.e. the main driving term of the freezing transition is the contribution $F_{\mathrm{DLVO}}$ to $F$. Freezing occurs at much lower packing fractions than that of the hard-sphere freezing transition [34], since the thick double layers, at the low salt concentrations under consideration here, give rise to a much larger effective diameter, and hence to a larger effective packing fraction, than the actual ones [23,35]. The 'pocket' of gas-liquid coexistence, which does not intersect the freezing line in any of the three phase diagrams of figure 1 , is not due to $F_{\mathrm{DLVO}}$ but instead is due to the volume term $F_{0}$ in the total free energy $F$. The reason is that the one-component system without attractive interactions, such as determines $F_{\mathrm{DLVO}}$, does not possess the cohesive energy required to stabilize a liquid phase. In contrast, the two electrostatic contributions to $F_{0}$, given by the last two terms of equation (7), do describe such cohesive energy as will be discussed in more detail in section 4 . The pocket of gas-liquid coexistence that results from $F_{0}$ was also found in reference [23] for dielectric constants, and hence values of $\lambda_{B}$, appropriate to aqueous suspensions. Here we show the systematic shrinking of this pocket as the diameter increases, i.e. as the surface charge density decreases. The two critical points that enclose the regime of gas-liquid coexistence coalesce when $D=D_{c}$, where the critical diameter $D_{c}$ is slightly larger than $380 \mathrm{~nm}$. The value of $D_{c}$ depends, of course, on the specific numerical values of $Z$ and $\lambda_{B}$. For $D>D_{c}$ there is no gas-liquid coexistence, and the single critical point at $D=D_{c}$ is called a hypercritical or bicritical point [36]. For decreasing $D<D_{c}$, at fixed $Z$, the gas-liquid pocket increases as the upper and lower critical points move apart in the $\eta-n_{s}$ plane. Eventually, at sufficiently small $D$ or high surface charge density, the G-L phase boundary intersects with that of the F-FCC transition. Such an intersection gives rise to $\mathrm{G}-\mathrm{L}-\mathrm{FCC}$ triple points.

In figure 2 we show an example of a phase diagram with such triple points. The diameter of the particles is $D=350 \mathrm{~nm}$ as in figure 1(b), but now the colloidal charge number is $Z=750$ instead of $Z=500$. The solvent is, as before, taken to be ethanol, with $\lambda_{B}=2.3 \mathrm{~nm}$. Figure 2(a) shows the gas-liquid upper critical point $(\times)$ at $\eta \simeq 0.04$ and $n_{s} \simeq 7 \mu \mathrm{M}$. At somewhat lower salt concentrations, $n_{s} \simeq 3-5 \mu \mathrm{M}$, we find three coexisting phases, i.e. a triplepoint $(\triangle)$. The triple-point gas phase is of extremely low packing fraction, $\eta<10^{-6}$, whereas the triple-point liquid and solid phases have $\eta \simeq 0.09$ and $\eta \simeq 0.11$, respectively. At salt concentrations below the triple point the colloidal liquid phase is no longer thermodynamically stable, and we find only coexistence between an extremely low-density gas and the solid. The trend of the packing fraction of the melting line decreasing with decreasing $n_{s}$ continues in this regime of $n_{s}$. Now the packing fraction of the gas-solid (evaporation) line decreases smoothly with decreasing $n_{s}$ until extremely low values, $\eta \simeq 0.007$ and $n_{s} \simeq 0.1 \mu \mathrm{M}$, are reached. This regime is shown in detail in figure 2(b). Perhaps surprisingly, we find another set of simultaneously coexisting gas, liquid, and solid phases $(\triangle)$ at $n_{s} \simeq 0.22-0.15 \mu \mathrm{M}$. As in the case of the upper triple point, the lower triple-point gas phase is extremely dilute $\left(\eta<10^{-6}\right)$, 

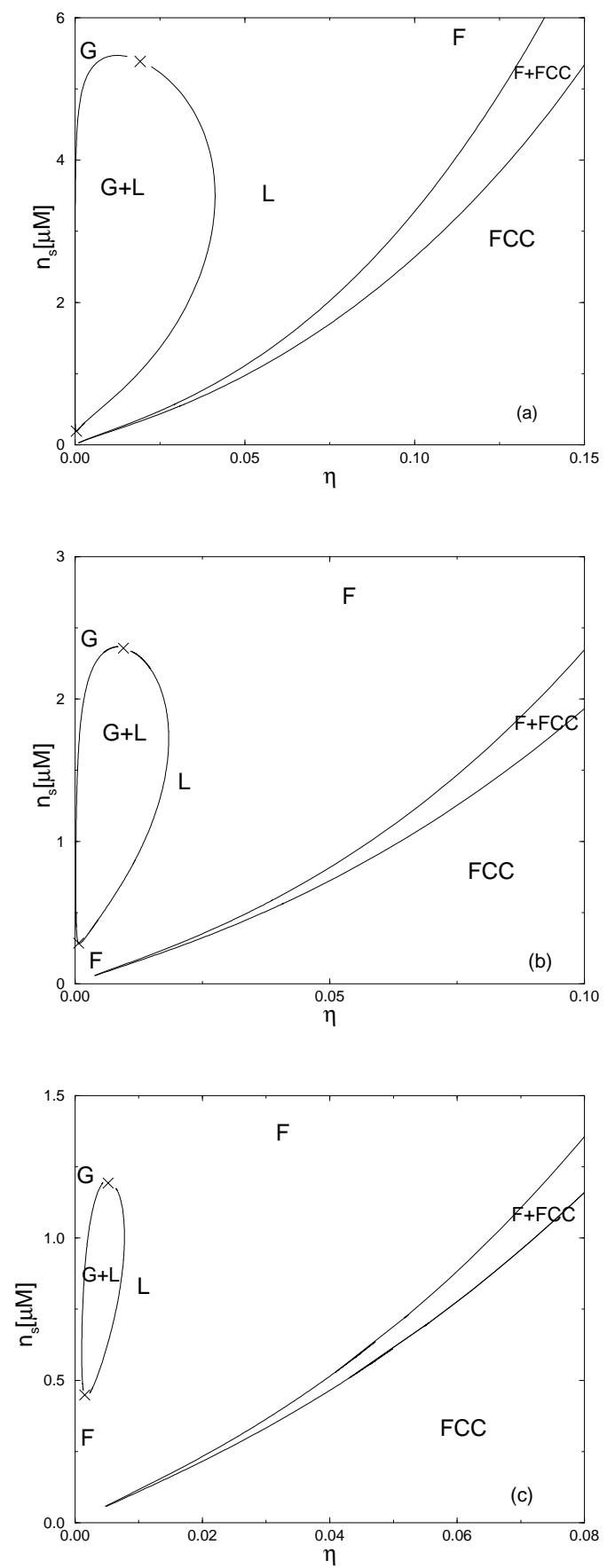

Figure 1. The phase diagram of a suspension of colloidal particles (packing fraction $\eta$ ) in a 1:1 electrolyte of density $n_{s}$ with the dielectric constant that of ethanol. The colloid charge number is $Z=500$, and the diameter is (a) $D=300$, (b) $D=350$, and (c) $D=380 \mathrm{~nm}$. We distinguish the fluid phase F, the crystalline FCC solid phase, and 'pockets' of coexisting gas $\mathrm{G}$ and liquid $\mathrm{L}$ with accompanying critical points $\times$. The $\mathrm{G}+\mathrm{L}$ two-phase regime decreases with increasing $D$, and disappears completely for $D>380 \mathrm{~nm}$. Note that the coexisting gas phase is extremely dilute, $\eta<10^{-6}$, for $0.2<n_{s}(\mu \mathrm{M})<4$ in (a). 


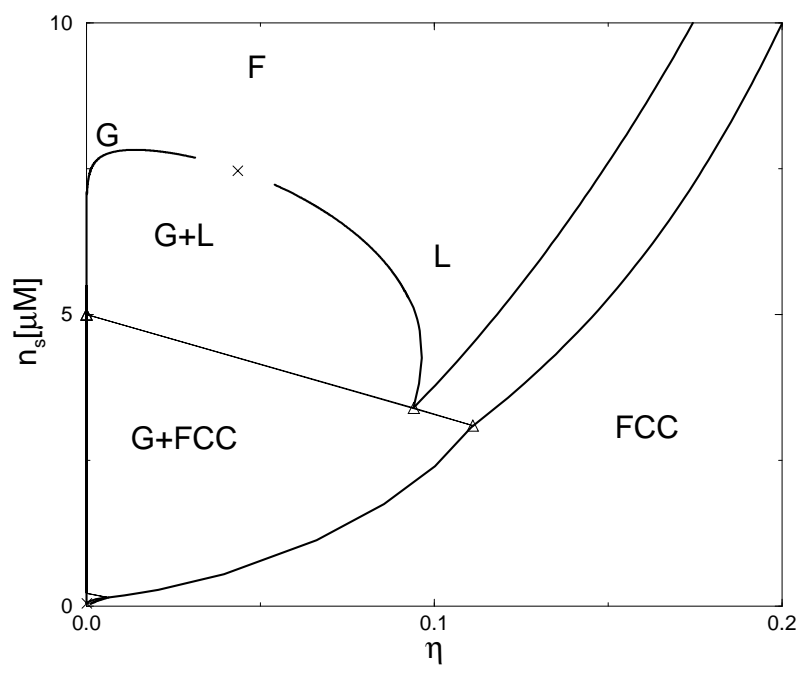

(a)

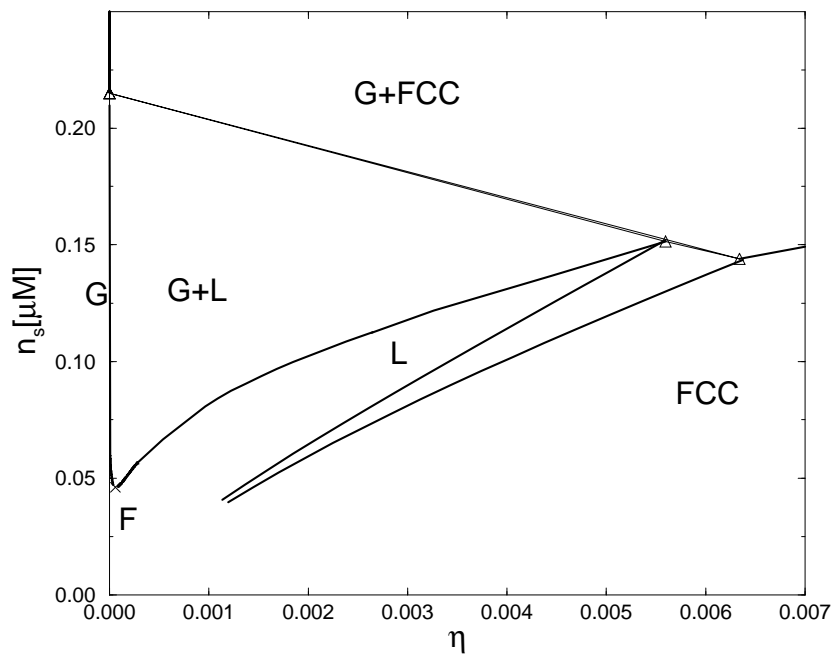

(b)

Figure 2. The phase diagram of a suspension of colloidal particles (packing fraction $\eta$ ) in a 1:1 electrolyte of density $n_{s}$ with the dielectric constant that of ethanol. The colloid charge number is $Z=750$, and the diameter is $D=350 \mathrm{~nm}$. The phases are denoted as in figure 1 , but here the regime of $\mathrm{G}+\mathrm{L}$ coexistence intersects the $\mathrm{F}+\mathrm{FCC}$ coexistence region to yield an upper and a lower triple point. Panel (a) displays the phase boundaries on an unexpanded scale. The $\Delta \mathrm{s}$ denote the upper triple-point gas, liquid, and solid phase, and $\times$ denotes the gas-liquid upper critical point. Panel (b) displays the results at very low salt concentrations. Here the $\Delta \mathrm{s}$ denote the lower triplepoint coexisting phases, and $\times$ denotes the lower gas-liquid critical point. Note the difference in scales between (a) and (b).

whereas the triple-point liquid and FCC solid phase have packing fractions $\eta \simeq 0.0055$ and 0.0065 , respectively. At $n_{s} \simeq 0.05 \mu \mathrm{M}$ and $\eta \simeq 10^{-4}$ we find the gas-liquid lower critical point. 
In principle we should also consider the possibility of a stable body-centred cubic (BCC) phase. Here we argue, however, that the $\mathrm{BCC}$ phase is not stable in the present parameter regime of interest; the fluid or the FCC phase is lower in free energy. A simple qualitative argument is obtained as follows. It is known from several studies of one-component Yukawa systems that the BCC phase can only be thermodynamically stable if $\xi<\xi_{m}$, where $\xi \equiv \kappa \rho^{-1 / 3}$ is a dimensionless combination of the Yukawa screening parameter $\kappa$ and the density $\rho$, and where $\xi_{m}$ is a temperature-dependent maximum that takes values of the order of $\xi_{m} \approx 1.72-5[37,38]$. Using equation (4) for $\kappa$ and setting $\rho=n_{c}$, it is easily verified that the condition $\xi<\xi_{m}$ gives rise to a necessary (but not sufficient) condition $n_{s}<n_{0} / Z^{2}$ to be satisfied if the $\mathrm{BCC}$ phase is possibly stable, with $n_{0}=\xi_{m}^{6} /\left(864 \pi^{3} \lambda_{B}^{3}\right)$. Using the Bjerrum length of ethanol, $\lambda_{B}=2.3 \mathrm{~nm}$, one then finds that $n_{0} \simeq 10^{-4} \mathrm{M}$ when $\xi_{m}=1.72$ and $n_{0} \simeq 0.1 \mathrm{M}$ when $\xi_{m}=5$. These values give rise to a salt concentration $n_{s}$ that is too low to allow for a stable $\mathrm{BCC}$ phase for the present colloid charges $Z$; the BCC phase is unlikely to be a stable phase in the regime of interest here.

The examples of phase separation shown in figures 1 and 2 do not involve the colloidal particles only, since the added salt fractionates simultaneously. In general the colloid-dilute phase has a higher added salt concentration than the colloid-dense phase with which it coexists, or, in other words, the tie lines that connect coexisting phases have a negative slope in the representation of figures 1 and 2 . It is very important, however, to realize that this does not imply that the total microion concentration in the colloid-dilute phase is higher than in the coexisting colloid-dense phase. In fact, the opposite is the case: the screening parameter $\kappa$ of the colloid-dense phase is always larger, due to the larger contribution from the counterions in the dense phase, than that of the coexisting colloid-dilute phase; see equation (4). This becomes explicit on considering the screening lengths $1 / \kappa$ at the two triple points of figure 2 . For the upper triple point we have $1 / \kappa \simeq 76,69$, and $68 \mathrm{~nm}$ in the gas, liquid, and solid phase, respectively, and for the lower triple point 366,306 , and $300 \mathrm{~nm}$. It will turn out to be a crucial ingredient of the phase separation mechanism that the double layers in the denser phases are more compressed than in the more dilute phases.

We emphasize that the phase diagrams in figures 1 and 2 result only from electrostatic and excluded-volume interactions in a three-component mixture; no long-ranged dispersion interactions between the colloidal particles have been included. In fact, one could envisage a situation where dispersive Van der Waals-London-Hamaker interactions are included, and give rise to gas-liquid coexistence (with a critical point) and a gas-solid coexistence (and a gas-liquid-solid triple point) at $n_{s} \simeq 0.01 \mathrm{M}$ without affecting the low-salt phase behaviour discussed here [6]. One would then have a phase diagram with three gas-liquid critical points, and three triple points [39]. Here, however, we focus on the low-salt regime, where the dispersion interactions can be safely ignored.

In section 4 we discuss the mechanism that drives the gas-liquid and gas-solid phase separation shown in figures 1 and 2, and in section 5 the mechanism that is responsible for the remixing and the existence of the lower critical point at extremely low salt concentrations.

\section{Demixing mechanism: connection with Debye-Hückel theory}

It is instructive to consider a limiting case that might give some extra insight into the mechanism that drives the phase transitions discussed in section 3 . We first reduce the three-component system under consideration to a two-component system by setting the added salt concentration $n_{s}=0$, leaving the (positive) counterions as the only microionic species. For convenience we also assume the colloids to be point particles, i.e. $R=0$. The limiting system is, therefore, equivalent to an asymmetric $1: Z$ electrolyte with species densities $Z n_{c}$ and $n_{c}$. Note that $\eta=0$ 
since $R=0$. A crude mean-field estimate of $F_{\mathrm{DLVO}}$, denoted $F_{\mathrm{DLVO}}^{\mathrm{MF}}$, is then

$$
\frac{F_{\mathrm{DLVO}}^{\mathrm{MF}}}{V}=k_{B} T n_{c}\left(\ln n_{c}-1\right)+\frac{1}{2} a n_{c}^{2}
$$

where $a$ is the integrated strength of the DLVO pair potential given in equation (6). With $R=0$ it follows that

$$
a=\left(Z^{2} e^{2} / \epsilon\right) \int \mathrm{d} r \exp [-\kappa r] / r=4 \pi Z^{2} e^{2} / \epsilon \kappa^{2} .
$$

This approximation is in the spirit of Van der Waals' theory for gas-liquid coexistence, in which half of the integrated strength of the attractions appears as the prefactor of the term in the free energy per unit volume that is quadratic in the density [2]. In the case of mutual attractions this contribution to the free energy is negative, but here we have $a>0$ because of the purely repulsive character of the pair interactions between the colloids. The first term of equation (9) is simply the ideal-gas contribution of the colloidal particles. Adding the estimate of equation (9) to the volume term of equation (7) yields, from equation (8), the sought-for mean-field approximation $F^{\mathrm{MF}}=F_{\mathrm{DLVO}}^{\mathrm{MF}}+F_{0}$ to $F$. It is easily checked that the final term of $F_{0}$ in equation (7) cancels the second term of $F_{\mathrm{DLVO}}^{\mathrm{MF}}$ in equation (9). Setting $\eta=0$ and recalling the ideal-gas contribution of the two species:

$$
F_{i d}=k_{B} T V\left[n_{c}\left(\ln n_{c}-1\right)+Z n_{c}\left(\ln Z n_{c}-1\right)\right]
$$

it follows that the excess (over the ideal) free-energy density is given by

$$
\frac{F^{\mathrm{MF}}-F_{i d}}{V k_{B} T}=-\frac{Z \kappa^{3}}{8 \pi} \propto-n_{c}^{3 / 2}
$$

since $\kappa^{2}=4 \pi e^{2} Z n_{c} / \epsilon k_{B} T$ now. For $Z=1$ this expression is remarkably similar, although not identical, to the Debye-Hückel expression for the excess free energy of a symmetric 1:1 electrolyte of point ions at density $n=n_{+}=n_{-}$, for which the right-hand side of equation (10) would have read $-\kappa_{D}^{3} / 12 \pi \propto-n^{3 / 2}$ with $\kappa_{D}^{2}=8 \pi e^{2} n / \epsilon k_{B} T$ [40,41]. Without the presence of the final term of $F_{0}$ we would not have obtained the cancellation of the term $a n_{c}^{2} / 2 \propto-n_{c}$. Then the lowest-order density correction on the right-hand side of equation (10) would have been $\mathcal{O}\left(n_{c}\right)$ instead of $\mathcal{O}\left(n_{c}^{3 / 2}\right)$. The existence of this final term and the resulting cancellation in the total mean-field free energy of the system is also discussed by Warren in reference [42].

The negative $\mathcal{O}\left(n^{3 / 2}\right)$ contribution to the free-energy density is well known, at least in the case of electrolytes, to give rise to a gas-liquid spinodal instability at low enough temperatures, i.e. at strong enough Coulomb coupling [41]. When the additional hard-core contributions to $F$ are taken into account properly, the instability gives rise to a 'Van der Waals loop', and hence to gas-liquid coexistence in the symmetric electrolyte [41]. The nature of the corresponding criticality has been hotly debated over the past few years, but simulation, experiment, and theory leave no doubt concerning the existence of such a critical point and, of course, gas-liquid coexistence below it $[41,43,44]$. Although the Debye-Hückel theory provides a poor account of the location of the phase boundary of the restricted primitive model of an electrolyte [41,44], it does contain the essential physics of the gas-liquid transition in these systems. Although the average local charge density vanishes by symmetry, every charge of the electrolyte is, by charge neutrality, surrounded by an oppositely charged 'cloud' of typical thickness $\lambda_{D}=1 / \kappa_{D}$. This correlation gives rise to an electrostatic (free-) energy contribution of the order of $-e^{2} / \epsilon \lambda_{D}$ per microion, or equivalently of order $-\kappa_{D}^{3} k_{B} T$ per unit volume. Thus, loosely speaking, a high-density liquid phase is stabilized by cohesive energy resulting from the closer average proximity of oppositely charged particles. A dilute gas phase is, as usual, stabilized by its high entropy. 
By identifying the correlation 'cloud' in the 1:1 electrolyte with the electric double layer in the colloidal suspension, the rather obvious connection between the two is now established. Indeed, the gas-liquid (and gas-solid) phase separation found in low-salt colloidal suspensions is driven mainly by that part of the volume term which reduces to the $\mathcal{O}\left(-n_{c}^{3 / 2}\right)$ term in equation (10), i.e. to the Debye-Hückel excess free energy of a 1:1 electrolyte (up to a numerical factor). The mechanism of the phase separation in the colloidal suspension is therefore similar to that which is well established in 1:1 electrolytes. In our language of colloidal suspensions we argue as follows. At high salt concentrations $n_{s} \gg Z n_{c}$, the double-layer thickness $1 / \kappa$ is essentially determined by the excess salt concentration $n_{s}$, independently of $n_{c}$; see the definition of $\kappa$ in equation (4). The typical separation between the charge in a double layer and 'its' colloidal particle is therefore essentially identical in dilute and dense colloid phases (provided that $Z n_{c} \ll n_{s}$ ). Consequently, there is no mechanism to provide an excess cohesive energy that stabilizes a liquid phase, i.e. that could balance the high entropy of a coexisting gas phase. The situation is different in the low-salt regime $n_{s} \leqslant Z n_{c}$, where $1 / \kappa$ is substantially smaller in a dense phase than in a dilute phase. Under these circumstances the typical distance between the surface charge of a colloidal particle and the charge in its surrounding double layer is smaller in a dense phase, and this can give rise to the required excess cohesive energy that stabilizes a liquid phase in coexistence with a gas. By analogy with the 1:1 electrolyte case, one should not speak of attractions between like-charged species in order to explain the phase separation-the colloids indeed repel each other via screened Coulomb interactions as is explicit in equation (6). Rather, the stable colloidal liquid phase is explained by the charge-neutrality-induced correlation effect whereby charged colloids are surrounded by an oppositely charged cloud of microions, i.e. whereby oppositely charged particles tend to be closer to each other, on average, than particles with the same sign of electric charge.

A connection between Debye-Hückel theory for simple electrolytes and fluid-fluid phase separation (or 'complex coacervation') in colloidal or polymeric suspensions was, in fact, already discussed in 1938 by Langmuir [45], and in more detail in 1956-1957 by Voorn and co-workers [46]. These authors made remarks along the lines discussed above, and identified the Debye-Hückel mechanism as the driving force behind the phase separation in these suspensions. It is our impression, however, that they did not make explicit connection between the Debye-Hückel theory and the effective one-component DLVO theory of colloidal suspensions [47]. Such a connection is, in principle, present in recent work by Levin et al, although their expression for the colloid-charge-cloud free-energy density does not reduce to a Debye-Hückel-like $\mathcal{O}\left(-n_{c}^{3 / 2}\right)$ term in the limit of a symmetric electrolyte of point ions [29]. In contrast, the present theory does establish this connection directly, via the volume term $F_{0}$. There is, however, an important quantitative difference between the present description and the earlier work of Langmuir and Voorn. This difference is also why the limiting case expressed in equation (10) does not reproduce the exact numerical prefactor of the Debye-Hückel limiting law. Whereas all charge-carrying species contribute to the screening in the Debye-Hückel theory for simple electrolytes and in the treatments of references [45,46], only the microions screen in the present theoretical description. In other words, a symmetry that is present in the Debye-Hückel theory is lost here; the colloidal particles are treated at a level different from the microions. More specifically, the density functional that underlies the present study does take into account the colloid-microion correlations-the microions experience the external potential of the colloids and form double layers around them - but the functional neglects the microion-microion correlations. Clearly, these correlations would give rise to an additional free-energy contribution at the Debye-Hückel level. It was argued in reference [25], however, 
that the colloid-microion correlations are far more important than the microion-microion correlations in the case of extreme charge asymmetry, and certainly in the regime of low added salt concentrations of interest here. Given the generally successful description of highsalt colloidal suspensions by the DLVO theory, in which the colloids do not contribute to the screening either, we expect the present semi-quantitative connection with Debye-Hückel theory to be sufficient to explain the main features of the low-salt behaviour of suspensions of highly charged colloidal particles. Nevertheless, it would be interesting to construct a theory that interpolates exactly between the symmetric 1:1 electrolyte and the extremely asymmetric colloidal suspensions. Such a theory should, amongst other things, answer the question of how large the colloidal parameters $Z$ and $D$ must be before the colloidal particles cease to contribute to the screening [39]. We leave this as an open problem.

\section{The mechanism of remixing}

Above, we argued that the gas-liquid and gas-solid phase separations, which occur at sufficiently low salt concentrations, are driven by the balance of the high entropy of the microions in the relatively expanded double layers of the dilute gas phase and the low electrostatic energy of the more compressed double layers of the dense liquid or solid phase. This demixing mechanism becomes stronger for lower salt concentrations, and therefore does not explain the existence, at even lower salt concentrations, of the lower critical and triple points shown in figures 1 and 2 . The competing mechanism required to remix at extremely low salt concentration is provided by the increasing range $1 / \kappa$, for decreasing $n_{s}$, of the screened Coulomb repulsions in the DLVO pair potential. The resulting free-energy contribution to $F_{\text {DLVO }}$ in the dense liquid or solid phase therefore increases with decreasing $n_{s}$ at fixed $n_{c}$. This explains, qualitatively, the slope of the melting and evaporation lines in figures 1 and 2 , as well as the tendency of the regime of gas-liquid and gas-solid coexistence to shrink at extremely low salt concentrations.

\section{Summary}

We have calculated the total Helmholtz free energy $F$ of a model of a charged colloidal suspension with added salt. By treating the electrostatics at the Debye-Hückel (or linearized Poisson-Boltzmann) level, we found that $F=F_{\mathrm{DLVO}}+F_{0}$. Here $F_{\mathrm{DLVO}}$ is the free energy of a one-component system of dressed colloids interacting via a pairwise, screened Coulomb (DLVO) repulsive potential, and $F_{0}$ is a volume term describing ideal-gas, excluded-volume, and electrostatic contributions of the microions. The cohesive electrostatic free energy (arising from the attraction between each negative colloid and its oppositely charged double layer) which enters $F_{0}$ gives rise to gas-liquid and gas-solid coexistence at low salt concentrations. This is despite the fact that the colloid-colloid DLVO interaction (and the corresponding freeenergy contribution $F_{\text {DLVO }}$ ) consists of repulsive contributions only.

We presented phase diagrams for several suspensions of charged colloidal particles with added monovalent salt in ethanol. At intermediate colloidal surface charge density, these exhibit a pocket of gas-liquid coexistence accompanied by an upper and a lower critical point. Below a certain critical total charge at a given colloid diameter, or above a certain critical diameter for a given total charge, there is no gas-liquid coexistence in the phase diagram. The disappearance of the gas-liquid coexistence regime upon decreasing surface charge density proceeds by the approach and final coalescence of the upper and lower critical point into a hypercritical or bicritical point. When the surface charge density is sufficiently large, the 
regime of gas-liquid coexistence is large enough to intersect the melting and freezing lines. This gives rise to an upper and a lower triple point in the phase diagram of highly charged colloids. The salt concentrations of the upper triple point are in the experimentally observable regime of a few micromoles per litre; those of the lower triple point are at least an order of magnitude smaller and therefore probably experimentally inaccessible.

The present theory provides a straightforward, semi-quantitative explanation for the experimental observations of gas-liquid and gas-solid coexistence in low-salt suspensions. Unlike in other recent approaches, the well-established DLVO theory for the pair interactions between the colloidal particles does not need to be abandoned in favour of a theory that describes long-ranged electrostatic colloid-colloid attractions. Rather, we noted that the total free energy $F$ of the suspension is not equal to $F_{\text {DLVO }}$ but involves the additional term $F_{0}$ that drives low-salt phase transitions. In addition, the physics contained within $F_{0}$ provides a direct connection between the Debye-Hückel theory for simple electrolytes and the DLVO theory for colloidal suspensions. It appears that the recent experimental observations of gas-liquid and gas-solid coexistence in low-salt suspensions of charged colloids have not been connected to the well-known gas-liquid transition in simple electrolytes. We believe that the theory discussed here and in references [22,23] provides this connection, and in that sense it links two well-known classical linearized Poisson-Boltzmann theories.

\section{Acknowledgments}

It is a pleasure to thank M Dijkstra, J-P Hansen, H N W Lekkerkerker, J Th G Overbeek, A P Philipse, R P Sear, P B Warren, and N B Wilding for sharing their insights in stimulating discussions. This work was supported by TMR grant ERBFMBICT971869 and by the EPSRC Liquid Matter Network.

\section{References}

[1] van der Waals J D 1873 PhD Thesis Hoogeschool te Leiden translated in

Lebowitz J L and Rowlinson J S (ed) 1988 Studies in Statistical Mechanics vol 14 (Amsterdam: Elsevier/NorthHolland)

[2] Hansen J-P and McDonald I R 1988 Theory of Simple Liquids 2nd edn (London: Academic)

[3] Derjaguin B and Landau L 1941 Acta Phys. Chim. URSS 14633

[4] Verwey E J W and Overbeek J Th G 1948 Theory of the Stability of Lyotropic Colloids (Amsterdam: Elsevier)

[5] Hamaker H C 1937 Physica 41058

[6] Victor J M and Hansen J-P 1985 Trans. Faraday Soc. II 8143

[7] See e.g.

Hansen J-P 1995 Phase Transitions in Complex Fluids ed M Baus, L F Rull and J P Rijckaert (Dordrecht: Kluwer)

[8] Ito K, Yoshida H and Ise N 1994 Science $\mathbf{2 6 3} 66$

Tata B V R, Yamahara E, Rajamani P V and Ise N 1997 Phys. Rev. Lett. 782660

[9] Tata B V R, Rajalakshmi M and Arora A K 1992 Phys. Rev. Lett. 693778 Palberg T and Würth M 1994 Phys. Rev. Lett. 72786

[10] Ise N, Obuko T, Sugimura M, Ito K and Nolte H J 1983 J. Chem. Phys. 78536 Ise N and Smalley M V 1994 Phys. Rev. B 5016722

[11] Larsen A E and Grier D G 1997 Nature 385230

[12] Sogami I 1983 Phys. Lett. A 96199

[13] Sogami I and Ise N 1984 J. Chem. Phys. 816329

[14] Tata B V R and Ise N 1998 Phys. Rev. E 582237

[15] Overbeek J Th G 1987 J. Chem. Phys. 874406

Sogami I S, Shinohara T and Smalley M V 1991 Mol. Phys. 74599

Sogami I S, Shinohara T and Smalley M V 1992 Mol. Phys. 761 
Overbeek J Th G 1993 Mol. Phys. 80685

Smalley M V and Sogami I S 1995 Mol. Phys. 85869

Schmitz K S 1996 Langmuir 123828

Ise N and Yoshida H 1996 Acc. Chem. Res. 293

[16] Woodward C E 1988 J. Chem. Phys. 895140

[17] Stevens M J and Robbins M O 1990 Europhys. Lett. 1281

Spalla O and Belloni L 1995 Phys. Rev. Lett. 742515

Ray J and Manning G S 1994 Langmuir 102450

Ha B Y and Liu A J 1997 Phys. Rev. Lett. 791289

Podgornik R and Parsegian V A 1998 Phys. Rev. Lett. 801560

Levin Y 1999 Physica A 265432

[18] Netz R R and Orland H 1999 Europhys. Lett. 45726

[19] Brilliantov N 1993 Phys. Rev. E 484536

[20] Stevens M J, Falk M L and Robbins M O 1996 J. Chem. Phys. 1045209

[21] Allahyarov E, D’Amico I D and Löwen H 1998 Phys. Rev. Lett. 811334

[22] van Roij R and Hansen J-P 1997 Phys. Rev. Lett. 793082

[23] van Roij R, Dijkstra M and Hansen J-P 1999 Phys. Rev. E 592010

[24] Evans R and Hasegawa M 1981 J. Phys. C: Solid State Phys. 145225

[25] Löwen H, Hansen J-P and Madden P A 1993 J. Chem. Phys. 983275

[26] Evans R 1979 Adv. Phys. 28143

[27] The microion densities that enter the definition of $\kappa$ in a linearized Poisson-Boltzmann theory are those at the point about which it is linearized. In the present case this point is the homogeneous density distribution whereas it is the zero of the electrostatic potential in much of the classical work. This difference can, perhaps, be traced back to the present use of density functional theory, which has the density distribution as its main object of manipulation, whereas the classical Poisson-Boltzmann equation has the potential as the natural variable.

[28] Grimson M J and Silbert M 1991 Mol. Phys. 74397 Graf H and Löwen H 1998 Phys. Rev. E 575744

[29] Levin Y, Barbosa M C and Tamashiro M N 1998 Europhys. Lett. 41123

[30] Lekkerkerker H N W and Stroobants A 1993 Physica A 195387 Dijkstra M, van Roij R and Evans R 1999 Phys. Rev. E 595744

[31] Borukhov I, Andelman D and Orland H 1997 Phys. Rev. Lett. 79435

[32] Shih W H and Stroud D 1983 J. Chem. Phys. 796254 Shih W Y, Aksay I A and Kikuchi R 1987 J. Chem. Phys. 865127

[33] Handbook of Chemistry and Physics 1957 77th edn, ed D R Lide (Boca Raton, FL: Chemical Rubber Company Press)

[34] Wood W W and Jacobson J D 1957 J. Chem. Phys. 271207 Alder B J and Wainwright T E 1957 J. Chem. Phys. 271208

[35] Wadati M and Toda M 1972 J. Phys. Soc. Japan 321147

[36] Sear R P, Warren P B and Wilding N B 1999 private communications

[37] Robbins M O, Kremer K and Grest G S 1988 J. Chem. Phys. 883286

[38] Meijer E J and Frenkel D 1991 J. Chem. Phys. 942269

[39] Warren P B 1999 private communications

[40] Debye P W and Hückel E 1923 Phys. Z. 24185

[41] Fisher M E and Levin Y 1993 Phys. Rev. Lett. 713826 Fisher M E 1994 J. Stat Phys. 751 and references therein

[42] Warren P B 1997 J. Physique II 7343

[43] See e.g. Pitzer K S 1995 J. Phys. Chem. 9913070 Kleemeier M, Wiegand S, Schröer W and Weingärtner H 1999 J. Chem. Phys. 1103085 and references therein

[44] See e.g. Caillol J M, Levesque D and Weis J J 1996 Phys. Rev. Lett. 774039 and references therein

[45] Langmuir I 1938 J. Chem. Phys. 6873

[46] Voorn M J 1956 PhD thesis University of Utrecht A summary of this thesis is contained in Michaeli J, Overbeek J Th G and Voorn M J 1957 J. Polym. Sci. 23443

[47] Of course, Langmuir's work [45] predates the DLVO theory, so he could not make a direct connection with the DLVO theory. 